\title{
TicTimer Web: software for measuring tic suppression
}

\section{remotely [version 1; peer review: 1 approved, 1 approved with}

\section{reservations]}

\author{
Jonathan K. Black (D1, Jonathan M. Koller2,3, Kevin J. Black (D)2-5
}

${ }^{1}$ Department of Mechanical Engineering, Brigham Young University, Provo, Utah, 84602, USA ${ }^{2}$ Department of Psychiatry, Washington University in St. Louis, St. Louis, Missouri, 63110, USA ${ }^{3}$ Department of Radiology, Washington University in St. Louis, St. Louis, Missouri, 63110, USA ${ }^{4}$ Department of Neurology, Washington University in St. Louis, St. Louis, Missouri, 63110, USA

${ }^{5}$ Department of Neuroscience, Washington University in St. Louis, St. Louis, Missouri, 63110, USA

V1 First published: 22 Oct 2020, 9:1264

https://doi.org/10.12688/f1000research.26347.1

Latest published: 03 Mar 2021, 9:1264

https://doi.org/10.12688/f1000research.26347.2

\begin{abstract}
Woods and Himle developed a standardized tic suppression paradigm (TSP) for the experimental setting, to quantify the effects of intentional tic suppression in Tourette syndrome. We previously provided a computer program to facilitate recording tic occurrence and to automate reward delivery during the several experimental conditions of the TSP. The present article describes a web-based program that performs the same functions. Implementing this program on the web allows research sessions to be performed remotely, in tandem with a video calling program. Relevant data for each session, such as the timing of tics and dispensed rewards, are stored in plain text files for later analysis. Expected applications include research on Tourette syndrome and related disorders.
\end{abstract}

\section{Keywords}

tc disorders, Tourette syndrome, reward, reinforcement (psychology), software

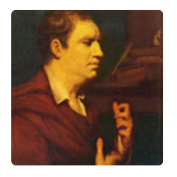

This article is included in the Tics collection.

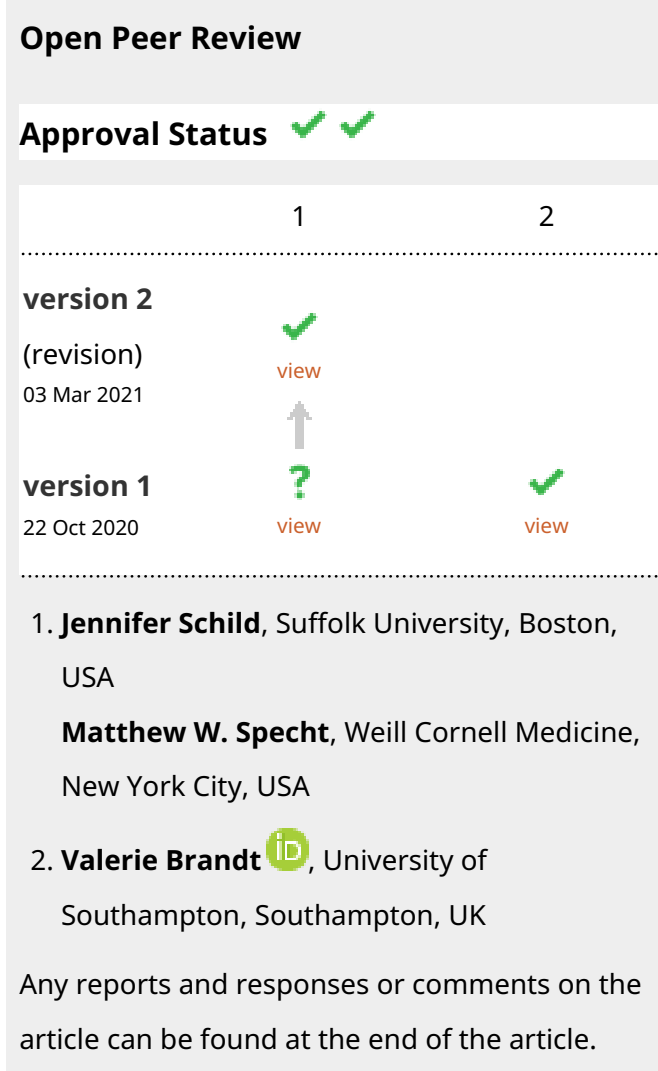


Corresponding authors: Jonathan K. Black (jonathan.knight.black@gmail.com), Kevin J. Black (kevin@wustl.edu)

Author roles: Black JK: Methodology, Software, Writing - Original Draft Preparation, Writing - Review \& Editing; Koller JM:

Methodology, Software, Writing - Review \& Editing; Black KJ: Conceptualization, Funding Acquisition, Project Administration,

Supervision, Writing - Original Draft Preparation, Writing - Review \& Editing

Competing interests: No competing interests were disclosed.

Grant information: Software development and manuscript preparation were funded in part by the U.S. National Institutes of Health (NIH), grant number R01MH104030.

The funders had no role in study design, data collection and analysis, decision to publish, or preparation of the manuscript.

Copyright: $\odot 2020$ Black JK et al. This is an open access article distributed under the terms of the Creative Commons Attribution License, which permits unrestricted use, distribution, and reproduction in any medium, provided the original work is properly cited.

How to cite this article: Black JK, Koller JM and Black KJ. TicTimer Web: software for measuring tic suppression remotely [version 1; peer review: 1 approved, 1 approved with reservations] F1000Research 2020, 9:1264 https://doi.org/10.12688/f1000research.26347.1

First published: 22 Oct 2020, 9:1264 https://doi.org/10.12688/f1000research.26347.1 


\section{Introduction}

In a previous article ${ }^{1}$ we mentioned the tic suppression paradigm (TSP) developed by Woods and Himle for use in the experimental setting to demonstrate and quantify the effects of intentional tic suppression on tic frequency in Tourette syndrome (TS) and other tic disorders ${ }^{2-6}$. In this paradigm, each participant is observed during several experimental conditions: usually baseline and differential reinforcement of zero-rate ticcing (DRO), and sometimes also verbal instruction to suppress tics and/or noncontingent reinforcement (NCR).

In the course of conducting a longitudinal study of children with Provisional Tic Disorder ${ }^{7}$, we found that tic suppression is seen within the first few months after a child's first tic ${ }^{8}$. We also found that the TSP required substantial investigator effort, so we wrote a simple program to facilitate record keeping and reward delivery during research sessions ${ }^{1}$. It required an expert observer to press a button to record each tic observed, and in the DRO and NCR conditions it delivered reward tokens at the appropriate times by connecting to a relay module and a token dispenser box. This software improved convenience for the investigator and accuracy of record-keeping.

Because of enforced social distancing in 2020, the need arose for sessions to be performed remotely. A video calling program allowed us to observe the subjects, but we still needed a way to deliver rewards during the DRO and NCR conditions of the TSP. Previously, we had created a web-based program called TicTrainer for behavioral therapy ${ }^{9}$, and we decided to expand the functionality of that program so it could be used for TSP research. We present the software here ${ }^{10}$ to facilitate its use by others.

\section{Methods}

Implementation

TicTimer $\mathrm{Web}^{10}$ uses the node.js server that was made for TicTrainer. It adopts that program's structure, with user accounts for research subjects and an admin account for the rater. Details on account registration, data storage, and logging on were described previously ${ }^{9}$.

A new field was added to user accounts so that a research ID (different from the ID used to $\log$ on) can be set for research subjects.

Sessions for TicTimer Web use separate but simultaneous connections to a server from a "user" and from a rater. TSP DRO sessions deliver rewards after every 10 -s tic-free interval. To deliver rewards at the appropriate times, the user page checks in with the server periodically to see if it has been 10 seconds since the last tic was signaled by the rater. If it has not, the server responds with the time remaining until the next reward is due, and the user page uses that number, adjusted for the lag time of the round trip, to schedule when to check back with the server. Using this method, reward timing is synchronized so that awards are delivered usually within $50 \mathrm{~ms}$ of the target time. The previous, in-person version of TicTimer used a token dispenser box to automatically deliver rewards at the appropriate times. In an attempt to approximate the user experience of the physical token dispenser, TicTimer Web delivers rewards by displaying coin images on the subject's screen along with a chime sound.

At the end of each session, a summary is generated and appended at the bottom of the session log file, which is then archived with the date and time of the session in the filename.

\section{Operation}

Setup. First, node.js is installed on the server. We used an Amazon EC2 instance, but the program can operate on any computer with node.js (e.g. a laptop).

The researcher and subject need only a modern web browser to interface with TicTimer Web once the server is running. The browser must support JavaScript and HTML5. We have tested TicTimer Web with current versions of Chrome and Edge.

To perform sessions with TicTimer Web, the researcher creates a user account if needed, then if desired uses the admin interface to assign the user a research ID number to identify the subject for later data analysis.

Use. The researcher and subject sign in on their respective TicTimer session pages and the rater begins the session by selecting one of the four experimental conditions.

During a session, the rater watches the subject. We have used a separate video calling program for this observation, but a video camera or one-way mirror could be used for in-person visits. The rater records any observed tics by immediately pressing the "Tic Detected" button, the spacebar, or the letter " $T$ ". If the session type includes rewards (DRO and NCR), they are dispensed appropriately. The session ends after the predetermined duration, or when the rater presses "End Session," or when either rater or subject closes their browser window early.

For the NCR condition, the rater first chooses a log file previously created with the current subject, and rewards are delivered to the user at the same times (relative to the session start) that they were delivered in the specified session.

Archived session log files can be downloaded from the admin interface, or they can be copied over directly from the server itself.

\section{Use cases}

The video file (Extended data, Supplementary File 1) ${ }^{11}$ demonstrates the operation of TicTimer Web ${ }^{10}$ from a researcher's perspective. The sessions performed here were test sessions with no human subjects being observed. Extended data, Supplementary Files $2-4^{11}$ are the session log files created in that video. 


\section{Conclusions}

The TicTimer $\mathrm{Web}^{10}$ program allows for remote implementation of the TSP, while maintaining the benefits of earlier versions of the software ${ }^{1}$ : ease and accuracy of record keeping and automated reward delivery. TicTimer Web, while designed for our purposes in tic disorder research, may find other uses. The most obvious of these may be for research on traditional habit disorders; for instance, hair pulling and skin picking appear in the "Obsessive-compulsive and related disorders" section of DSM- $5^{12}$. The most obvious application to the clinical setting may be in documenting suppression ability before and after treatment. Another potential future modification would be to add machine detection of tics, e.g. by online video analysis, surface EMG or accelerometry; such an improvement would be quite welcome but is difficult to reduce to practice.

\section{Data availability}

Underlying data

All data underlying the results are available as part of the article and no additional source data are required.

\section{Extended data}

Zenodo: TicTimer Web: software for measuring tic suppression remotely: Supplementary Files. http://doi.org/10.5281/zenodo. $4023134^{11}$.
This project contains the following extended data:

- ttw_demo.mp4. (Supplementary File 1: Video Demonstration of Operation. A video demonstrating how to operate TicTimer Web from the researcher's perspective.)

- au5_20200622-153043_baseline.ttsd. (Supplementary File 2: Sample Log, baseline. Log file for the baseline session performed during the video demonstration.)

- au5_20200622-153125_DRZ.ttsd. (Supplementary File 3: Sample Log, DRO. Log file for the DRO session performed during the video demonstration.)

- au5_20200622-153214_NCR.ttsd. (Supplementary File 4: Sample Log, NCR. Log file for the NCR session performed during the video demonstration.)

License: MIT License.

\section{Software availability}

The source code for TicTimer Web is available at: https://github. com/jonkb/TicTrainer-node.

Archived source code at time of publication: https://doi. org/10.5281/zenodo. $3990474^{10}$.

License: MIT License.
1. Black JK, Koller JM, Black KJ: TicTimer software for measuring tic suppression [version 2; peer review: 2 approved]. F1000Res. 2017; 6: 1560. PubMed Abstract | Publisher Full Text | Free Full Text

2. Woods DW, Himle MB: Creating tic suppression: comparing the effects of verbal instruction to differential reinforcement. J Appl Behav Anal. 2004; 37(3): 417-20.

PubMed Abstract | Publisher Full Text | Free Full Text

3. Himle MB, Woods DW: An experimental evaluation of tic suppression and the tic rebound effect. Behav Res Ther. 2005; 43(11): 1443-51.

PubMed Abstract | Publisher Full Text

4. Himle MB, Woods DW, Bunaciu L: Evaluating the role of contingency in differentially reinforced tic suppression. J Appl Behav Anal. 2008; 41 (2): 285-9. PubMed Abstract | Publisher Full Text | Free Full Text

5. Lyon G], Samar SM, Conelea C, et al.: Testing tic suppression: comparing the effects of dexmethylphenidate to no medication in children and adolescents with attention-deficit/hyperactivity disorder and Tourette's disorder.J Child Adolesc Psychopharmacol. 2010; 20(4): 283-9. PubMed Abstract | Publisher Full Text | Free Full Text

6. Conelea CA, Wellen B, Woods DW, et al.: Patterns and Predictors of Tic Suppressibility in Youth With Tic Disorders. Front Psychiatry. 2018; 9: 188. PubMed Abstract | Publisher Full Text | Free Full Text
7. Black KJ, Kim S, Schlaggar BL, et al.: The New Tics study: A novel approach to pathophysiology and cause of tic disorders. J Psychiatr Brain Sci. 2020; 5: e200012.

PubMed Abstract | Publisher Full Text | Free Full Text

8. Greene DJ, Koller JM, Robichaux-Viehoever A, et al.: Reward enhances tic suppression in children within months of tic disorder onset. Dev Cogn Neurosci. 2015: 11: 65-74.

PubMed Abstract | Publisher Full Text | Free Full Text

9. Black JK, Black KJ: Software for web-based tic suppression training [version 2; peer review: 3 approved]. F1000Res. 2018; 6: 2150.

PubMed Abstract | Publisher Full Text | Free Full Text

10. Black JK, Black KJ: TicTrainer and TicTimer Web v4.0.2 (Version 4.0.2). Zenodo. 2020.

http://www.doi.org/10.5281/zenodo.3990474

11. Black JK, Koller JM, Black KJ: TicTimer Web: software for measuring tic suppression remotely: Supplementary Files. F1000research. Zenodo. 2020 http://www.doi.org/10.5281/zenodo.4023134

12. American Psychiatric Association: Obsessive-Compulsive and Related Disorders. In: Diagnostic and Statistical Manual of Mental Disorders. Fifth Edition. Arlington, VA: American Psychiatric Association; 2013. Publisher Full Text 


\section{Open Peer Review}

\section{Current Peer Review Status:}

\section{Version 1}

Reviewer Report 17 February 2021

https://doi.org/10.5256/f1000research.29084.r77056

(C) 2021 Brandt V. This is an open access peer review report distributed under the terms of the Creative Commons Attribution License, which permits unrestricted use, distribution, and reproduction in any medium, provided the original work is properly cited.

\section{Valerie Brandt}

Department of Psychology, Centre for Innovation in Mental Health, University of Southampton, Southampton, UK

The report describes the development of TicTimer Web, a nifty software that can be used to record tics, tic timings, and distribute reinforcement tokens during online experiments are therapeutic sessions. The authors describe that they adapted the TicTrainer programme to an online version that allows patients to login to a user account and therapists / tic raters to login as administrators. User and rater are then connected to the server simultaneously. The tic rater can record tics via button press and the software stores the timing of that tic. After every tic-free 10 second interval, the software delivers a reward. The software also delivers a summary at the end of the session. In order for the rater to watch tics, a separate programme, or a webcam can be used. This allows an accurate reward delivery, even remotely.

The source code of TicTimer is available on GitHub, the link is provided in the manuscript. Furthermore, an explanatory video shows how TicTimer can be used. The video is simple and clear and provides step-by-step explanations. Examples of demo files are also provided on Zenodo.

The manuscript is clearly written, the software is demonstrated in a video, examples and the source code are provided.

I have no suggestions for improvement.

Is the rationale for developing the new software tool clearly explained?

Yes

Is the description of the software tool technically sound?

Yes

Are sufficient details of the code, methods and analysis (if applicable) provided to allow replication of the software development and its use by others? 
Yes

Is sufficient information provided to allow interpretation of the expected output datasets and any results generated using the tool?

Yes

Are the conclusions about the tool and its performance adequately supported by the findings presented in the article?

Yes

Competing Interests: No competing interests were disclosed.

Reviewer Expertise: Tic disorders.

I confirm that I have read this submission and believe that I have an appropriate level of expertise to confirm that it is of an acceptable scientific standard.

Author Response ( F1000Research Advisory Board Member) 22 Feb 2021

Kevin J Black

We thank Prof. Brandt for her kind comments.

Competing Interests: No competing interests were disclosed.

Reviewer Report 20 January 2021

https://doi.org/10.5256/f1000research.29084.r73543

(C) 2021 Specht $\mathbf{M}$ et al. This is an open access peer review report distributed under the terms of the Creative Commons Attribution License, which permits unrestricted use, distribution, and reproduction in any medium, provided the original work is properly cited.

Jennifer Schild

Suffolk University, Boston, MA, USA

Matthew W. Specht

Weill Cornell Medicine, New York City, NY, USA

This paper describes a timely and valuable tool for assessing tic suppression remotely in clinical and research settings. This web-based program is based on Woods and Himle's standardized tic suppression paradigm (TSP) and performs the same function as a computer program previously developed by the authors to record tics and partially automate delivery of rewards. This paper is particularly useful during this time in which remote data collection for research is relevant and necessary, and it provides a helpful overview of a program that can be easily understood by other tic researchers to apply in their own research. The paper could benefit from elaboration in multiple areas to help readers better understand the broader relevance of TSP as well as the 
research utility of TicTimer Web. Elaboration in the introduction section, as well as a more detailed description of potential applications of this software and how this software helps to overcome previous limitations of the TSP are needed. We recommend that this article be revised and resubmitted. Below are some suggestions for the authors to consider.

1. Please provide a clearer, more detailed explanation of the broad importance of TSP and related concepts in the introduction section. This will be helpful for readers who are less familiar with tic suppression to understand what insights this paradigm have yielded and how this software program could be beneficial to future investigation. For example, the TSP is described as requiring "substantial investigator effort," but it is not clear what exactly is entailed. Those who used the old tic-detector boxes know how laborious running a participant was, but those less familiar will not appreciate the leap forward TicTimer Web provides.

2. A short description of the TicTrainer web-based program is also needed in the introduction section. This will be helpful for orienting the reader to the new program that is described in this article, especially considering how similar this older program is to TicTimer Web.

3. In the methods section, there is interchangeable usage of the term "reward" and "award." The word "awards" in paragraph 3 of the implementation subsection of the methods should be replaced with "rewards."

4. In the methods section, elaboration is needed on what each experimental condition is (e.g., differential reinforcement of zero-rate ticcing (DRO), noncontingent reinforcement (NCR). Alternatively, this can be elaborated upon in the introduction section as it is also mentioned there.

5. A separate section before the conclusion discussing the strengths and future applications of this software would be useful. This is somewhat addressed in the conclusion section, but further elaboration in a devoted section would do a better job of highlighting the potential of this software program.

6. Next steps: It would be helpful to provide another sentence or two regarding the potential benefits of using artificial intelligence (AI) and machine learning (ML) to do this job as well as barriers to taking this approach.

Overall, this paper provides a concise and useful overview of a web-based program for remote implementation of the TSP. With some clarification in the areas highlighted above, this paper offers a useful contribution to tic research and is especially applicable given the current relevance of remote methods for data collection. We strongly encourage the authors to revise and resubmit this manuscript for consideration.

Is the rationale for developing the new software tool clearly explained? Partly

Is the description of the software tool technically sound? Yes

Are sufficient details of the code, methods and analysis (if applicable) provided to allow replication of the software development and its use by others? 
Yes

Is sufficient information provided to allow interpretation of the expected output datasets and any results generated using the tool?

Partly

Are the conclusions about the tool and its performance adequately supported by the findings presented in the article?

Partly

Competing Interests: No competing interests were disclosed.

Reviewer Expertise: I have 15 years of research regarding Tourette's disorder and have utilized the tic detector paradigm in my own research.

We confirm that we have read this submission and believe that we have an appropriate level of expertise to confirm that it is of an acceptable scientific standard, however we have significant reservations, as outlined above.

Author Response ( F1000Research Advisory Board Member ) 22 Feb 2021

Kevin J Black

We thank Ms. Schild and Dr. Specht for their thoughtful review, and will edit the report as they suggest.

Competing Interests: No competing interests were disclosed.

Author Response ( F1000Research Advisory Board Member) 24 Feb 2021

Kevin J Black

Author responses are below, marked by the > sign and bold face, intercalated between sections of the original review.

APPROVED WITH RESERVATIONS

This paper describes a timely and valuable tool for assessing tic suppression remotely in clinical and research settings. This web-based program is based on Woods and Himle's standardized tic suppression paradigm (TSP) and performs the same function as a computer program previously developed by the authors to record tics and partially automate delivery of rewards. This paper is particularly useful during this time in which remote data collection for research is relevant and necessary, and it provides a helpful overview of a program that can be easily understood by other tic researchers to apply in their own research.

$>$ Thank you for these positive comments. 
The paper could benefit from elaboration in multiple areas to help readers better understand the broader relevance of TSP as well as the research utility of TicTimer Web. Elaboration in the introduction section, as well as a more detailed description of potential applications of this software and how this software helps to overcome previous limitations of the TSP are needed. We recommend that this article be revised and resubmitted. Below are some suggestions for the authors to consider.

1. Please provide a clearer, more detailed explanation of the broad importance of TSP and related concepts in the introduction section. This will be helpful for readers who are less familiar with tic suppression to understand what insights this paradigm have yielded and how this software program could be beneficial to future investigation. For example, the TSP is described as requiring "substantial investigator effort," but it is not clear what exactly is entailed. Those who used the old tic-detector boxes know how laborious running a participant was, but those less familiar will not appreciate the leap forward TicTimer Web provides.

$>$ Done (first 2 paragraphs).

1. A short description of the TicTrainer web-based program is also needed in the introduction section. This will be helpful for orienting the reader to the new program that is described in this article, especially considering how similar this older program is to TicTimer Web.

$>$ Done (last paragraph of Intro).

1. In the methods section, there is interchangeable usage of the term "reward" and "award." The word "awards" in paragraph 3 of the implementation subsection of the methods should be replaced with "rewards."

$>$ Thank you. Corrected.

1. In the methods section, elaboration is needed on what each experimental condition is (e.g., differential reinforcement of zero-rate ticcing (DRO), noncontingent reinforcement (NCR). Alternatively, this can be elaborated upon in the introduction section as it is also mentioned there.

$>$ Done (in first paragraph of Intro).

1. A separate section before the conclusion discussing the strengths and future applications of this software would be useful. This is somewhat addressed in the conclusion section, but further elaboration in a devoted section would do a better job of highlighting the potential of this software program.

$>$ I elaborate on these in the first paragraph of the revised Conclusions section.

1. Next steps: It would be helpful to provide another sentence or two regarding the potential benefits of using artificial intelligence (AI) and machine learning (ML) to do this job as well as barriers to taking this approach.

$>$ Done (last paragraph of Conclusions).

Overall, this paper provides a concise and useful overview of a web-based program for remote implementation of the TSP. With some clarification in the areas highlighted above, this paper offers a useful contribution to tic research and is especially applicable given the 
current relevance of remote methods for data collection. We strongly encourage the authors to revise and resubmit this manuscript for consideration.

\section{$>$ Thank you.}

Competing Interests: None

The benefits of publishing with F1000Research:

- Your article is published within days, with no editorial bias

- You can publish traditional articles, null/negative results, case reports, data notes and more

- The peer review process is transparent and collaborative

- Your article is indexed in PubMed after passing peer review

- Dedicated customer support at every stage

For pre-submission enquiries, contact research@f1000.com 